TAIWANESE JOURNAL OF MATHEMATICS

Vol. 1, No. 1, pp. 59-63, March 1997

\title{
A NOTE ON THE ADMISSIBILITY OF P-VALUE FOR THE ONE-SIDED HYPOTHESIS TEST IN THE NEGATIVE BINOMIAL MODEL
}

\author{
Jine-Phone Chou
}

\begin{abstract}
Let $\mathrm{X}$ be a random variable with negative binomial density

$$
f(x \mid \theta)=\frac{\Gamma(x+r)}{\Gamma(x+1) \Gamma(r)} \theta^{x}(1-\theta)^{r},
$$

where $x=0,1,2, \cdots, 0<\theta<1, r>0$. For the hypothesis testing problem

$$
H_{0}: \theta \leq \theta_{0} \quad \text { versus } \quad H_{1}: \theta>\theta_{0}
$$

based on observing $\mathrm{X}=x$, where $\theta_{0}$ is specified, we consider it as an estimation problem within a decision-theoretic framework. We prove the admissibility of estimator $p(x)=P_{\theta_{0}}(X \geq x)$, the $p$-value, for estimating the accuracy of the test, $\mathbf{1}_{\left(0, \theta_{0}\right)}(\theta)$, under the squared error loss.
\end{abstract}

\section{INTRODUCTION}

For a random vector $\mathrm{X}$ with the parameter space $\Theta$, Hwang, Casella, Robert, Wells, and Farrell (1992) from decision-theorectic approach did the hypothesis testing problem

$$
H_{0}: \theta \in \Theta_{0} \quad \text { versus } \quad H_{1}: \theta \in \Theta_{0}^{c}
$$

based on observing $\mathrm{X}=x$, where $\Theta_{0}$ is a specified subset of $\Theta$ in the following framework. We want to know the viability of the set specified by $H_{0}$ by estimating the parameter $\mathbf{1}_{\Theta_{0}}(\theta)$ (where $\mathbf{1}_{A}(\cdot)$ denotes the indicator of set $\mathrm{A}$ ), and we consider the parameter $\mathbf{1}_{\Theta_{0}}(\theta)$ to measure the accuracy of the test. In

Received January 11, 1996.

Communicated by I.-S. Chang.

1991 Mathematics Subject Classification: 62F03, 62A99, 62C07, $62 \mathrm{C} 15$.

Key words and phrases: Hypothesis testing, squared error loss, admissibility, prior, Bayes estimator, the accuracy of a test, $p$-value. 
one word we do the hypothesis testing problem by estimating the parameter $\mathbf{1}_{\Theta_{0}}(\theta)$, the accuracy of the test, and the performance of an estimator, say $\delta(x)$, is evaluated by some loss function $L(\theta, \delta)=d\left(\mathbf{1}_{\Theta_{0}}(\theta)-\delta(x)\right)$, where $d(t)$ is minimum at $t=0$, nondecreasing for $t>0$ and nonincreasing for $t<0$. In this note we do a hypothesis testing problem in the same decision-theoretic framework.

The definition of the $p$-value of a hypothesis test follows Lehmann's (1986) through the note, and we consider the admissibility of the $p$-value for the problem of estimating the accuracy of the one-sided testing problem under the squared error loss,

$$
H_{0}: \theta \leq \theta_{0} \quad \text { versus } H_{1}: \theta>\theta_{0}
$$

where $\theta_{0}$ is a specified point. There are many criticisms raised at the $p$-value to be as a measure of evidence against the null hypotheses for the hypothesis testing problem (see, e.g., Lindley (1957); Berger and Sellke (1987)). Specifically it is generally inadmissible for estimating the accuracy in a two-sided hypothesis testing problem (Hwang, et al., (1992)). However for the one-sided hypothesis testing problem of some random varibles with location parameter, symmetric density and monotone likelihood ratio, the $p$-value can be reconciled with the infimum of the Bayesian measure of evidence against the null hypotheses (Casella and Berger (1987)). Moreover for the problem (1.1) considered here, the $p$-value is admissble in many modles. For example if the randon variable $\mathrm{X}$ is from the model of normal $N(\theta, 1)$, or binomial $B(n, \theta)$, or Poisson $P(\theta)$, Hwang, et al., (1992) had proved the $p$-value is admissible, and in this note for $\mathrm{X}$ from the negative binomial model, $N B(r, \theta)$, we prove the $p$-value, $p(x)=P_{\theta_{0}}(X \geq x)$, does also have the admissibility property for estimating the accuracy of the problem (1.1).

\section{Result}

Let $\mathrm{X}$ be a random variable with negative binomial density

$$
f(x \mid \theta)=\frac{\Gamma(x+r)}{\Gamma(x+1) \Gamma(r)} \theta^{x}(1-\theta)^{r},
$$

where $x=0,1,2, \cdots, 0<\theta<1, r>0$. For the problem of estimating $\mathbf{1}_{\left(0, \theta_{0}\right)}(\theta)$, the accuracy of the one-sided hypothesis test (1.1), with loss function

$$
L(\theta, \delta)=\left(\delta(x)-\mathbf{1}_{\left(0, \theta_{0}\right)}(\theta)\right)^{2},
$$

where $\delta(x)$ is an estimator of $\mathbf{1}_{\left(0, \theta_{0}\right)}(\theta)$, we are going to prove that estimator $p(x)=P_{\theta_{0}}(X \geq x), x$ the observed value, is a generalized Bayes estimator with finite Bayes risk, and thus admissible. 
Theorem. Let $X$ be a random variable with the density (2.1). For the problem of estimating the accuracy of the one-sided hypothesis test $(1.1), \mathbf{1}_{\left(0, \theta_{0}\right)}(\theta)$, with the loss $(2.2)$, the $p$-value $p(x)=P_{\theta_{0}}(X \geq x)$ is an admissible estimator.

Proof. Choose an improper prior $\pi$ on the parameter space $\Theta=(0,1)$ with

$$
d \pi=\frac{1}{\theta(1-\theta)} \mathbf{1}_{(0,1)}(\theta) d \theta
$$

where $d \theta$ is the Lebesgue measure on R. First note that for any estimator $\delta(X)$, the posterior Bayes risk at $x=0$ is finite if and only if $\delta(x)=1$ at $x=0$. Since the estimator $p$-value $p(x)=1$ at $x=0$, then the $p$-value achieves the minimum posterior Bayes risk at $x=0$. For $x \geq 1$, the posterior density is

$$
g(\theta \mid x)=\frac{f(x \mid \theta) \pi(\theta)}{\int_{0}^{1} f(x \mid \theta) \pi(\theta) d \theta}=\frac{\Gamma(x+r)}{\Gamma(x) \Gamma(r)} \theta^{x-1}(1-\theta)^{r-1},
$$

and the Bayes estimator, say $\mathrm{B}(x)$, is the conditional expection of $\mathbf{1}_{\left(0, \theta_{0}\right)}(\theta), E_{\theta \mid x}$ $\mathbf{1}_{\left(0, \theta_{0}\right)}(\theta)$. By using the technique of changing variable in the integration and the binomial theorem, calculations give us

$$
\begin{aligned}
B(x) & =E_{\theta \mid x} \mathbf{1}_{\left(0, \theta_{0}\right)}(\theta) \\
& =\int_{0}^{\theta_{0}} \frac{\Gamma(x+r)}{\Gamma(x) \Gamma(r)} \theta^{x-1}(1-\theta)^{r-1} d \theta \\
& =1-\frac{\Gamma(x+r)}{\Gamma(x) \Gamma(r)} \int_{\theta_{0}}^{1}(\theta)^{x-1}(1-\theta)^{r-1} d \theta \\
& =1-\frac{\Gamma(x+r)}{\Gamma(x) \Gamma(r)} \int_{0}^{1-\theta_{0}}(1-\theta)^{x-1}(\theta)^{r-1} d \theta \\
& =1-\frac{\Gamma(x+r)}{\Gamma(x) \Gamma(r)} \int_{0}^{1}\left(1-\left(1-\theta_{0}\right) \theta\right)^{x-1}\left(1-\theta_{0}\right)^{r-1} \theta^{r-1}\left(1-\theta_{0}\right) d \theta \\
& =1-\left(1-\theta_{0}\right)^{r} \frac{\Gamma(x+r)}{\Gamma(x) \Gamma(r)} \int_{0}^{1}\left((1-\theta)+\theta_{0} \theta\right)^{x-1}(\theta)^{r-1} d \theta \\
& =1-\left(1-\theta_{0}\right)^{r} \frac{\Gamma(x+r)}{\Gamma(x) \Gamma(r)} \int_{0}^{1} \sum_{k=0}^{x-1}\left({ }^{x-1}\right)(1-\theta)^{x-1-k} \theta_{0}^{k} \theta^{k}(\theta)^{r-1} d \theta \\
& =1-\sum_{k=0}^{x-1}\left(1-\theta_{0}\right)^{r} \theta_{0}^{k} \frac{\Gamma(x+r)}{\Gamma(x) \Gamma(r)}\left({ }_{k}^{x-1}\right) \int_{0}^{1}(1-\theta)^{x-1-k} \theta^{k+r-1} d \theta
\end{aligned}
$$




$$
\begin{aligned}
B(x) & =1-\sum_{k=0}^{x-1}\left(1-\theta_{0}\right)^{r} \theta_{0}^{k} \frac{(k+r-1) !}{k !(r-1) !} \\
& =P_{\theta_{0}}(X \geq x) \\
& =p(x) .
\end{aligned}
$$

Therefore $p(x)$ is a generalized Bayes estimator and the theorem will be proved if the Bayes risk is finite. Consider the Bayed risk of $p(x), r(\pi, p)$. With $g(\theta \mid x)$ being given in (2.4) and $k_{1}$ denoting some constant,

$$
\begin{aligned}
r(\pi, p) & =\int \sum_{x=0}^{\infty}\left(\mathbf{1}_{\left(0, \theta_{0}\right)}(\theta)-p(x)\right)^{2} f(x \mid \theta) d \pi \\
& =\int_{\theta_{0}}^{1} \theta^{-1}(1-\theta)^{r-1} d \theta+\sum_{x=1}^{\infty}\left\{\int_{0}^{1}\left(\mathbf{1}_{\left(0, \theta_{0}\right)}(\theta)-p(x)\right)^{2} g(\theta \mid x) d \theta\right\} \frac{1}{x} \\
& \leq k_{1}+\sum_{x=1}^{\infty}\left(p(x)-(p(x))^{2}\right) \frac{1}{x} .
\end{aligned}
$$

Since

$$
\begin{aligned}
p(x) & =\sum_{n=x}^{\infty} \frac{\Gamma(n+r)}{\Gamma(n+1) \Gamma(r)} \theta_{0}^{n}\left(1-\theta_{0}\right)^{r} \\
& =\theta_{0}^{x} \sum_{m=0}^{\infty} \frac{(m+x+r-1) \cdots(m+r)}{(m+x) \cdots(m+1)} \frac{\Gamma(m+r)}{\Gamma(m+1) \Gamma(r)} \theta_{0}^{m}\left(1-\theta_{0}\right)^{r} \\
& \leq \theta_{0}^{x} \sum_{m=0}^{\infty} k_{2} f\left(m \mid \theta_{0}\right)=k_{2} \theta_{0}^{x}
\end{aligned}
$$

where $k_{2}$ is some contant and $f$ is the density (2.1), hence $\sum_{x=1}^{\infty} p(x) \frac{1}{x}<\infty$. This together with the fact $(p(x))^{2} \leq p(x)$ imply the finite of $r(\pi, p)$.

Remark. For the hypothesis testing problem

$$
H_{0}: \theta \leq \theta_{0} \quad \text { versus } H_{1}: \theta>\theta_{0}
$$

based on observing $X=x$, where $X$ has the negative binomial density (2.1), the Bayesian measure of evidence given a prior distribution $\pi(\theta)$, is the probability that $H_{0}$ is true given $X=x$,

$$
P_{r}\left(H_{0} \mid x\right)=P_{r}\left(\theta \leq \theta_{0} \mid x\right)=\frac{\int_{0}^{\theta_{0}} f(x \mid \theta) d \pi(\theta)}{\int_{0}^{1} f(x \mid \theta) d \pi(\theta)},
$$

where $f$ is the density (2.1). Since $P_{r}\left(H_{0} \mid x\right)=E_{\theta \mid x} \mathbf{1}_{\left(0, \theta_{0}\right)}(\theta)$ and from the proof of theorem, we know that the $p$-value, $p(x)=P_{\theta_{0}}(X \geq x)$, a frequentist 
measure of evidence against $H_{0}$, is equal to the posterior probability of $H_{0}$ with the prior (2.3), a Bayesian measure of evidence against $H_{0}$.

\section{REFERENCES}

1. J. O. Berger and T. Sellke, Testing a point null hypothesis: The irreconcilability of $p$-values and evidence (with discussion), J. Amer. Statist. Assoc. 82 (1987), 112-139.

2. G. Casella and R. L. Berger, Reconciling Bayesian and Frequentist evidence in the one-sided testing problem (with discussion), J. Amer. Statist. Assoc. 82 (1987), 106-139.

3. E. L. Lehmann, Testing Statistical Hypothesis, 2nd ed. Wiley, New York, 1986.

4. D. V. Lindley, A statistics paradox, Biometrika 44 (1957), 187-192.

5. J. T. Hwang, G. Casella, C. Robert, M. T. Wells, and R. H. Farrell, Estimation of accuracy in testing, Ann. Statist. 20 (1992), 490-509.

Institute of Statistical Science, Academia Sinica

Taipei 11529, Taiwan 